\title{
Human pulmonary macrophages: the relationship of smoking to the presence of sea blue granules and surfactant turnover
}

\author{
PIERS N PLOWMAN* AND ROGER J FLEMANS $\dagger$ \\ From *Cambridge University and MRC Department of Clinical Oncology and Radiotherapeutics and \\ $\dagger$ Cambridge University Department of Haematological Medicine, The Medical School, Hills Road, \\ Cambridge, UK
}

SUMMARY Human pulmonary macrophages from heavy smokers usually (17/21 cases) contain sea blue inclusion material in the cytoplasm. In non-smokers, sea blue material is rarely evident (1/21 cases). The sea blue material observed has the same properties as sea blue material identified in other conditions (viz, Sudan black and PAS positivity and autofluorescence). Evidence is presented which suggests that pulmonary macrophages from smokers contain extra surfactant and that it is this phospholipid complex that accounts for the sea blue reaction observed.

In 1947, Moeschlin'1 described 'blue' histiocytes in the spleen of a patient with undiagnosed splenomegaly. In 1970, Silverstein et al. $^{2}$ reviewed nine cases with similar clinical features and coined the name 'syndrome of the sea blue histiocyte'. The underlying biochemical abnormality was excessive and abnormal storage of phospholipids and glycosphingolipids in the viscera. In all cases, large histiocytes (20-60 $\mu$ diameter) containing numerous bright blue granules (3-4 $\mu$ diameter) were seen in bone marrow aspirates using the Romanowsky stains. Jones et al. ${ }^{3}$ subsequently reported the clinical syndrome in siblings and postulated a hereditary disorder of lipid metabolism transmitted as an autosomal recessive trait. In both the cases reported in that communication chest radiographs demonstrated diffuse nodular densities in both lung fields.

In 1976, Parker et al. ${ }^{4}$ described a case of sea blue histiocytosis in spleen and marrow accompanying hyperlipidaemia (due to an increase in 'free' blood cholesterol, lecithin, and triglycerides). These authors concluded that sea blue staining histiocytes/ macrophages were markers of abnormal lipid metabolism.

The secondary nature of sea blue positivity in marrow macrophages (for example, in myeloid leukaemias) has recently been amplified in work from this centre. ${ }^{5}$

Received for publication 6 December 1979
In 1978, one of us (RJF) observed sea blue granules in human alveolar/pulmonary macrophages derived from a healthy person. Questions posed were: Did this person suffer from the primary syndrome of sea blue histiocytosis? Could the staining phenomenon be confined to this local peripheral tissue macrophage population-perhaps as a secondary phenomenon to some local pulmonary pathology? The present study was undertaken to answer these questions.

\section{Material and methods}

PATIENTS

Written consent was obtained from all bronchoscoped subjects. Healthy persons and patients (usually with bronchogenic carcinoma) were studied.

\section{ALVEOLAR LAVAGE}

Ringer lactate (Hartman's solution) lavages were performed by standard technique via an Olympus BFB3 bronchofiberscope or by lavage of freshly resected lung specimens.

\section{CYTOCHEMISTRY}

Leishman's stain, Perl's stain for free iron, the Sudan black B stain, and the PAS reaction were carried out by standard methods. ${ }^{6}$ Butyrate and dual esterase reactions were performed according to the methods described by Higgy et al., ${ }^{7}$ and acid phosphatase reaction by the method of $\mathrm{Li}$ et al. ${ }^{8}$ 


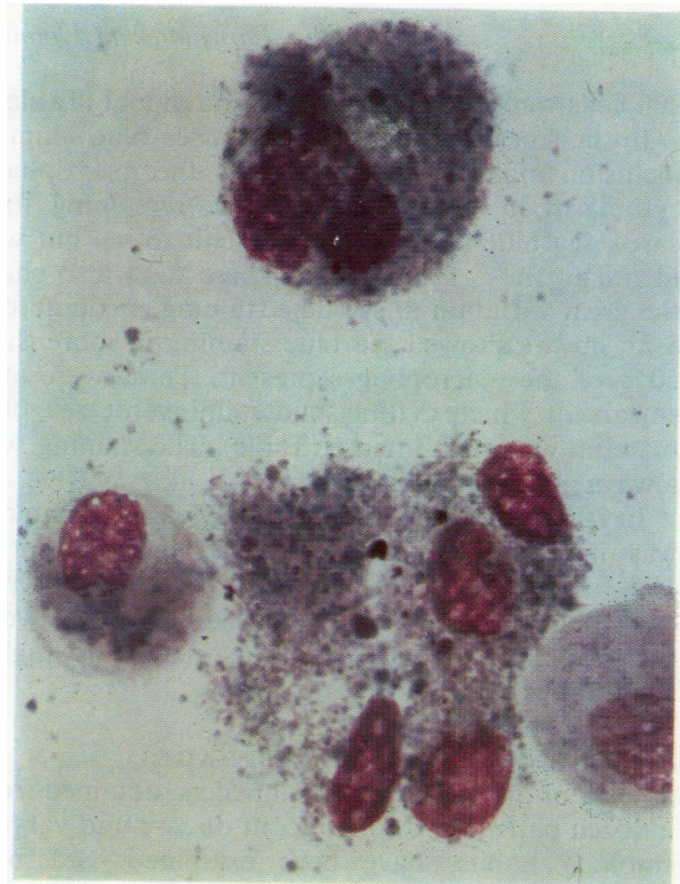

1

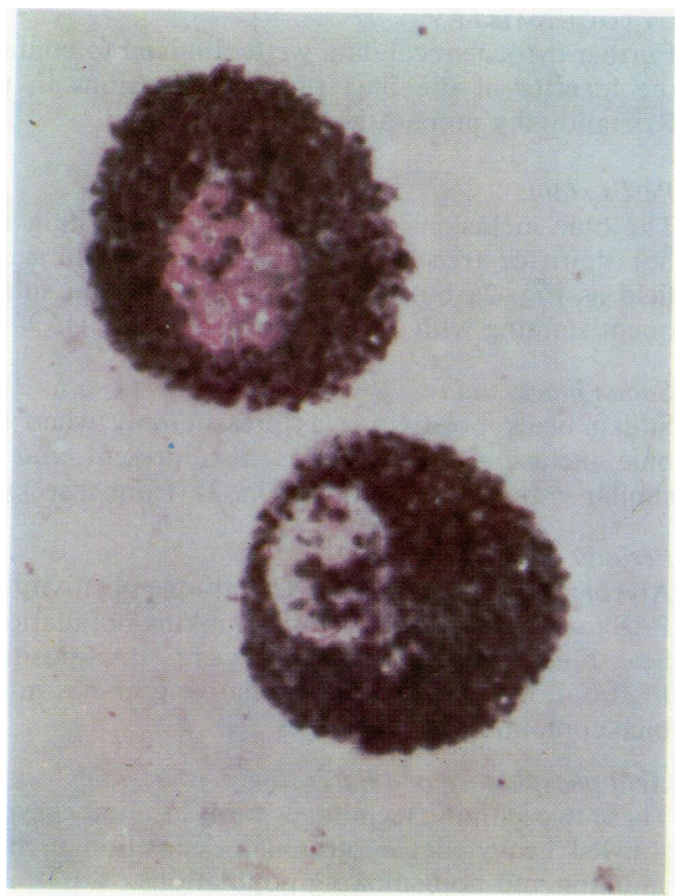

3

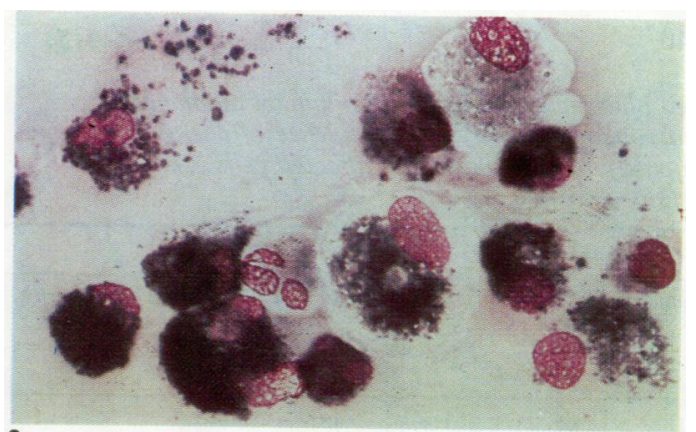

$2 \mathrm{a}$

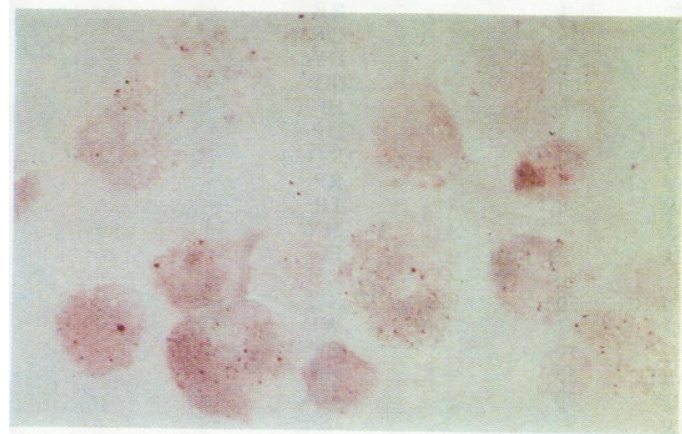

$2 b$

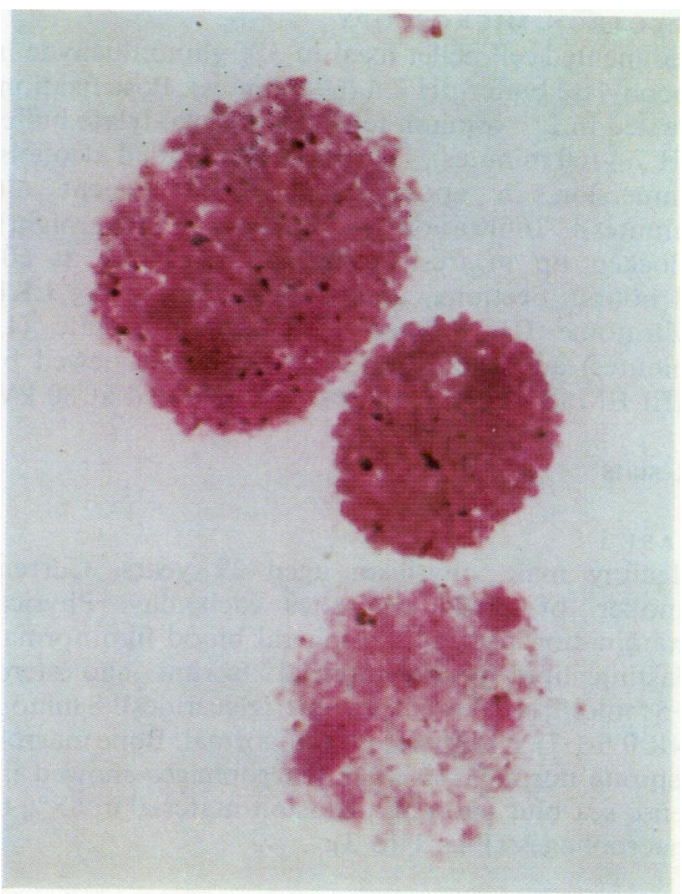

4

Fig. 1 Alveolar macrophages from case 1-Leishman's stain.

Fig. 2 (a) Alveolar macrophages from case 1-Leishman's stain. (b) The same field of cells as in Fig. $2 a$ after decolorisation and subsequent staining with Perl's stain.

Fig. 3 Alveolar macrophages from case 1-Sudan Black stain.

Fig. 4 Alveolar macrophages-PAS stain. 
Sea blue inclusion material in human pulmonary macrophages. Analysis of incidence of positive cells and intensity of staining within affected cells: smokers versus non-smokers

\begin{tabular}{|c|c|c|c|c|c|}
\hline \multicolumn{3}{|c|}{ Heavy smokers } & \multicolumn{3}{|c|}{ Non-smokers } \\
\hline Subject & $\begin{array}{l}\% \text { Cells } \\
\text { with sea } \\
\text { blue } \\
\text { material }\end{array}$ & $\begin{array}{l}\text { Intensity } \\
\text { of } \\
\text { staining }\end{array}$ & Subject & $\begin{array}{l}\% \text { Cells } \\
\text { with sea } \\
\text { blue } \\
\text { material }\end{array}$ & $\begin{array}{l}\text { Intensity } \\
\text { of } \\
\text { staining }\end{array}$ \\
\hline $\mathbf{P L}$ & 65 & +++ & JS & - & - \\
\hline $\mathbf{R P}$ & 32 & ++ & NP & - & - \\
\hline AC & 4 & $+t$ & RB & - & - \\
\hline $\mathbf{A R}$ & 76 & $+t+$ & GK & - & - \\
\hline HS & 5 & + & DW & - & - \\
\hline AB & 60 & $++t$ & MB & - & - \\
\hline GF & 10 & + & JB & - & - \\
\hline JR & 62 & $++t$ & DP & - & - \\
\hline HD & 50 & $++t$ & RD & - & - \\
\hline EW & - & \pm & $\mathbf{R P}$ & - & - \\
\hline VW & 8 & $+t$ & TR & - & - \\
\hline FR & - & \pm & MW & 15 & + \\
\hline KH & - & \pm & FC & - & - \\
\hline NB & 80 & $+t+$ & $\mathbf{X H}$ & - & - \\
\hline AMcK & 70 & $+t+$ & $\mathrm{XC}$ & - & - \\
\hline HG & 92 & +++ & WD & - & - \\
\hline FS & 10 & + & FN & - & - \\
\hline JMcI & 35 & ++ & HJ & - & - \\
\hline GC & 20 & ++ & CW & - & - \\
\hline SD & - & \pm & JS & - & - \\
\hline DW & 20 & $+t$ & DD & - & - \\
\hline
\end{tabular}

ELECTRON MICROSCOPY

Sedimented cell pellet fixed in $3 \%$ glutaraldehyde in cacodylate buffer pH 7.4 (60 minutes). Post fixation, treated in $2 \%$ osmium tetroxide in cacodylate buffer pH 7.4 (60 minutes). Dehydrated in graded alcohols. Immersion in epoxypropane link reagent (10 minutes). Infiltration in epoxyresin (overnight). Blocked up in fresh resin and hardened at $60^{\circ}$ (8 hours). Sections cut at $500^{\circ} \mathrm{A}$ thick using LKB Ultratome III. Stained with uranyl acetate (15 minutes) and lead citrate (10 minutes). Viewed by AEI EM6B electron microscope operated at $80 \mathrm{kV}$.

\section{Results}

CASE 1

Healthy male physician aged 29 years. Current smoker of 20-25 cigarettes each day. Physical examination normal. Peripheral blood film normal. Fasting lipid profile normal (serum cholesterol $4.5 \mathrm{mmol} / 1$, NR 3.6-6.2; serum triglycerides $0.8 \mathrm{mmo} / 1$, NR 0.6-1.7). Chest radiograph normal. Bone marrow aspirate normal. Alveolar macrophages showed intense sea blue staining inclusion material in $65 \%$ of macrophages (Figs 1 to 3 ).

CASES $1-42$

The sea blue staining reactions of alveolar macrophages derived from 21 heavy smokers (more than 20 cigarettes each day) have been compared with those of 21 non-smokers. Not less than
200 cells were scored from each individual (Table).

In the smears of heavy smokers, sea blue staining inclusion material was present in the macrophage cytoplasm in $17 / 21$ cases. In the remaining four cases, sea blue material was difficult to see but was identified in $<1 \%$ of cells. These data also show the great variation in sea blue staining encountered; $8 / 21$ smears showed sea blue staining in more than $50 \%$ of the macrophages present. These were also smears in which cytoplasmic staining for sea blue material was most intense (Table). The staining was always granular.

In 20/21 smears of non-smokers, sea blue material was undetectable, but in one case it was present in $15 \%$ of the cells. The intensity of staining of this individual's cells was weak. The difference between the smoking and non-smoking groups is highly significant $\left(\mathrm{P}<0.001\left(\chi^{2}\right.\right.$ test $\left.)\right)$.

\section{PLEURAL ASPIRATE MACROPHAGES}

Smears of pleural effusion cell pellets, obtained from a dozen patients with malignant disease and varying smoking habits, have been examined. Sea blue material has never been seen.

\section{CYTOCHEMISTRY}

Further cytochemical data were obtained to confirm the identity of the blue staining inclusions in the Romanowsky preparations.

\section{Perl's stain}

The blue inclusions on the Romanowsky stain did not stain for free iron. Figure $2 b$ shows the same field as Fig. 2a but after decolorisation and subsequent staining with Perl's stain (from case 1).

\section{Sudan black stain}

Sudan black positivity was present only when sea blue inclusion material was also present and of similar granular deposition (Fig. 3) (from case 1).

\section{Periodic acid Schiff stain}

Alveolar macrophages showed variable positivity for PAS in both smoking and non-smoking populations. Sea blue staining granules were PAS-positive. Figure 4 demonstrates PAS-positive granules in sea blue containing macrophages.

\section{Acid phosphatase and butyrate esterase stains}

These two staining techniques for lysosomal enzymes showed heavy, dense, granular deposition in both smoking and non-smoking populations.

\section{FLUORESCENCE}

Unstained macrophages from populations containing sea blue inclusion material showed autofluorescence under the ultraviolet microscope. 


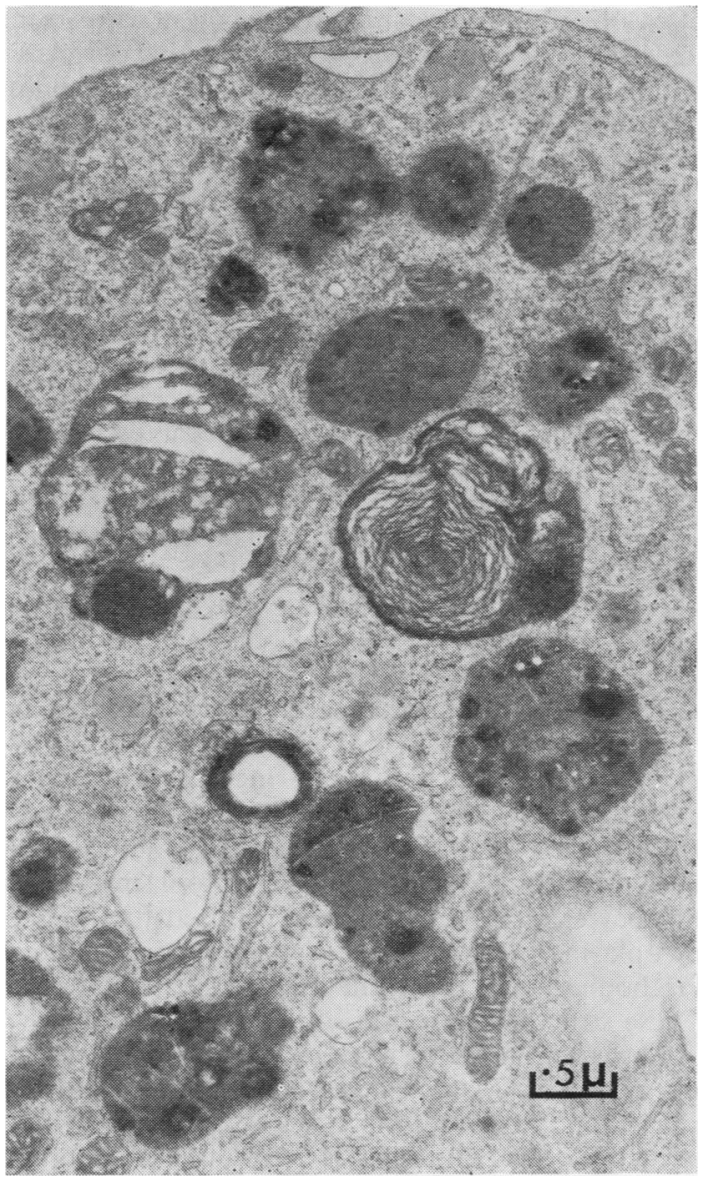

Fig. 5 Alveolar macrophage cytoplasmic inclusion'lamellar body' (electron microscope photo).

\section{ELECTRON MICROSCOPY}

Alveolar macrophages from populations containing sea blue inclusion material were examined by electron microscopy. 'Lamellar bodies' were an obvious feature in the cytoplasm of these cells (Figs 5 and 6). 'Fingerprints' were also observed (Fig. 7). Not enough non-smokers were studied for a conclusive comparison to be made.

Lamellar bodies appeared osmiophilic, but nonosmicated comparison preparations were technically difficult and results unsatisfactory.

\section{Discussion}

The present work demonstrates sea blue granules confined to one peripheral tissue macrophage population (viz, lung macrophages) in an 'aberrant' but not necessarily diseased group of subjects (viz, smokers).

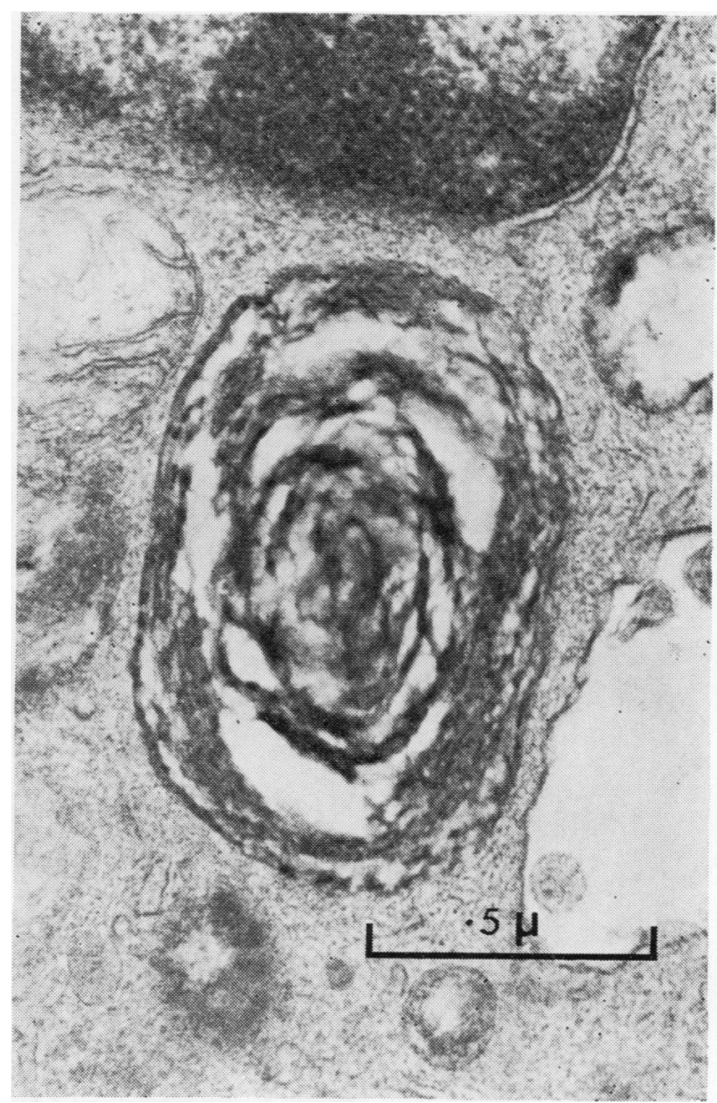

Fig. 6 Alveolar macrophage cytoplasmic inclusion'lamellar body' (electron microscope photo).

The biochemical abnormality in the previously described cases of 'sea blue histiocytosis' has been one of lipid metabolism. Phospholipids, often sphingomyelins, have been present in excess in these patients. ${ }^{2} 3$ Sphingomyelins are composed of a fatty acid, phosphoric acid, and both choline $\left(\mathrm{HOCH}_{2} \mathrm{CH}_{2} \stackrel{+}{\mathrm{N}}\left(\mathrm{CH}_{3}\right)_{3} \mathrm{O} \overline{\mathrm{H}}\right)$ and sphingosine. Choline forms the nitrogenous base for the lecithin family of phosphatides. ${ }^{9}$

Pulmonary surfactant is probably a mixutre of lipids of the lecithin family, and dipalmitoyl lecithin may be a lipid normally unique to the lung. ${ }^{10}$ Animal work suggests that pulmonary macrophages normally function in the uptake and hence turnover of surfactant. ${ }^{10} 11$

In 1972, Finley and Ladman ${ }^{12}$ reported a deficit in surfactant recovered in the lavage effluent after endobronchial lavage in smokers. The level of surfactant rapidly returned to normal after cessation of tobacco abuse. They found no qualitative 


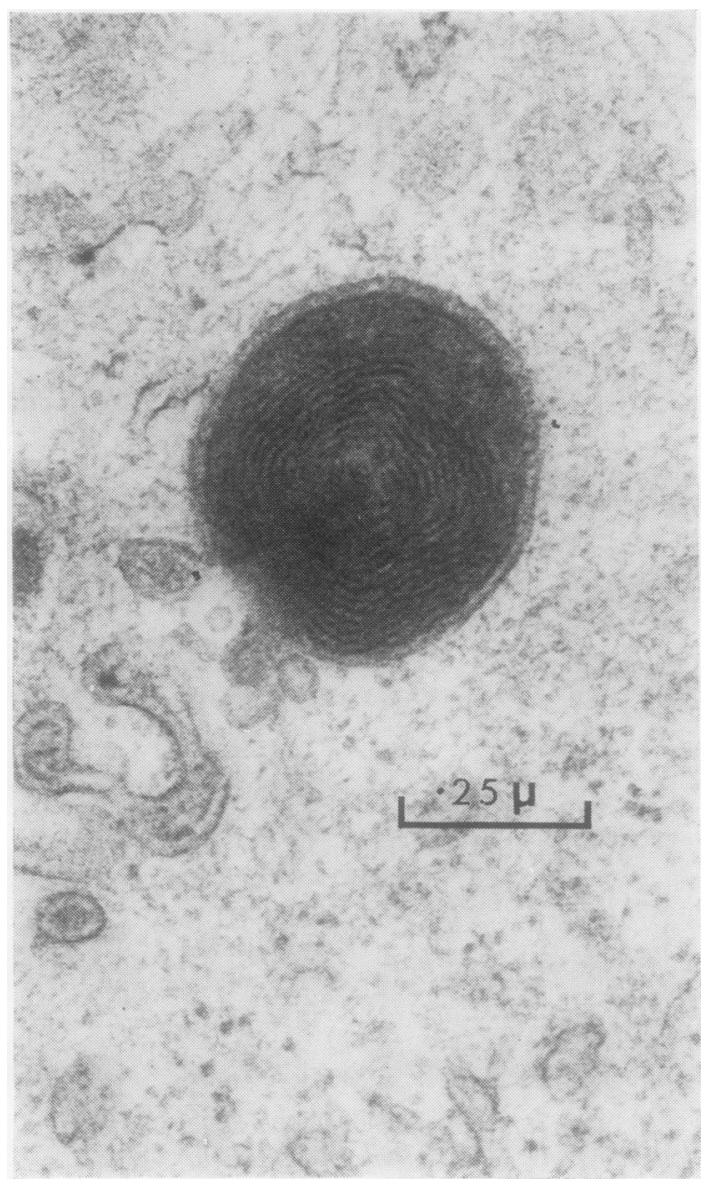

Fig. 7 Alveolar macrophage cytoplasmic inclusion'fingerprint' (electron microscope photo).

differences in phospholipid and neutral lipid species in supernatant or cell pellet in the two groups (smokers versus non-smokers). However, quantitative analysis of their data ${ }^{13}$ showed a sevenfold reduction in phosphatidylcholine (PC) content of supernatant and a more than twofold increase in the cell pellet. A reasonable conclusion was that smoking caused a shift in PC into the cells through both an increase in pulmonary macrophage numbers in smokers ${ }^{14}$ and possibly also increased ingestion by individual macrophages. This latter possibility is contentious, but evidence is accumulating to support this concept $^{14}$ (Plowman PN, in preparation).

The data presented here show sea blue inclusion material to be a prominent feature in the cytoplasm of alveolar macrophages from the majority of heavy smokers. The numbers of cells containing sea blue material varied widely, but in $8 / 21$ cases more than
$50 \%$ of cells were positive, the highest score being $92 \%$. The intensity of cytoplasmic staining correlated with the number of cells exhibiting the staining phenomenon.

In the non-smoking population only one person (who had been a non-smoker for two years and lived a mixed urban-rural existence) showed any sea blue material, and the score was comparatively low. If sea blue material was present in the other 20 cases examined, it was below the level detectable by light microscopy. The same was true of pleural fluid macrophages.

The identity of the blue inclusion material as the sea blue material described by Silverstein et al. ${ }^{2}$ was proved by other means. The cytochemistry data presented here confirm that the observed sea blue material is Sudan black positive, PAS positive, and autofluorescent-as described previously for sea blue material. ${ }^{31516}$ There can be no confusion now with ingested iron (Fig. 2).

The electron microscopic data demonstrated the 'lamellar bodies' described previously by Nicholls ${ }^{10}$ in her work on surfactant ingestion by rabbit alveolar macrophages and by Parker et al. ${ }^{4}$ in their work on sea blue histiocytosis. These authors also described 'fingerprints' in the cytoplasm of the histiocytes, and we have demonstrated these in the cytoplasm of affected alveolar macrophages. 'Lamellar bodies' may represent unravelled 'fingerprints'. These structures seemed osmiophilic.

The granular deposition of the sea blue material on light microscopy suggested a lysosomal disposition, and the electron microscopic data demonstrated the structures just described to be membranewrapped. The cytochemical stains for two lysosomal enzymes (viz, acid phosphatase and butyrate esterase) also showed coarse granular staining. The intensity of staining of the macrophages was the same in both smokers and non-smokers for these lysosomal enzymes. This is perhaps unexpected.

The chemical similarity of surfactant to the phospholipids sequestered in the histiocytes of the patients described by Silverstein et al..$^{2}$ suggested to us that intracellular surfactant might be the sea blue inclusion material observed here. This possibility is strengthened by the data of Finley and Ladman, ${ }^{12}$ who showed that there is a shift of PC into pulmonary macrophages in smokers.

The relevance of these observations to the chronic lung diseases of smokers is unknown.

We are indebted to $\mathrm{Mr}$ John Emmines for the excellence of the electron microscopic data. We thank Professor NM Bleehen and Professor FGJ Hayhoe for their support and advice, and Mrs Rosemary Leech for typing the manuscript. 


\section{References}

${ }^{1}$ Moeschlin S. Die Milzpunktion: Technik, diagnostische und hämatologische Ergebnisse. Basle: B Schwabe, 1947;37-8.

2 Silverstein MN, Ellefson RD, Ahern EJ. The syndrome of the sea-blue histiocyte. $N$ Engl J Med 1970;282:1-4.

${ }^{3}$ Jones B, Gilbert EF, Zugibe FT, Thompson H. Sea blue histiocyte disease in siblings. Lancet 1970;ii:73-5.

4 Parker AC, Bain AD, Brydon WG, et al. Sea-blue histiocytosis associated with hyperlipidaemia. J Clin Pathol 1976;29:634-8.

${ }^{5}$ Hayhoe FGJ, Flemans RJ, Cowling DC. Acquired lipidosis of marrow macrophages. J Clin Pathol 1979; $32: 420-8$.

${ }^{6}$ Hayhoe FGJ, Flemans RJ. An Atlas of Haematological Cytology. Wolfe Publications Ltd, 1969;316-7.

${ }^{7}$ Higgy KE, Burns GF, Hayhoe FGJ. Discrimination of B, $T$ and null lymphocytes by esterase cytochemistry. Scand J Haematol 1977;18:437-48.

${ }^{8}$ Li CY, Yam LT, Lam KW. Studies of acid phosphatase isoenzymes in human leukocytes: demonstration of isoenzyme cell specificity. $J$ Histochem Cytochem 1970; 18:901-10.

${ }^{9}$ Fieser LF, Fieser M. Organic Chemistry, 3rd ed. New York: Rheinhold Publishing, 1956;488-90.
10 Nicholls BA. Normal rabbit alveolar macrophages. I. The phagocytosis of tubular myelin. J Exp Med 1976;144: 906-19.

11 Naimark A. Cellular dynamics and lipid metabolism in the lung. Fed Proc 1973;32:1967-9.

${ }^{12}$ Finley TN, Ladman AJ. Low yield of pulmonary surfactant in cigarette smokers. $N$ Engl J Med 1972;286:223-7.

${ }^{13}$ Clements JA. Smoking and pulmonary surfactant. $N$ Engl $J$ Med 1972;286:261-2.

${ }^{14}$ Harris JO, Swensen EW, Johnson JE 3rd. Human alveolar macrophages: comparison of phagocytic ability, glucose utilisation and ultrastructure in smokers and nonsmokers. J Clin Invest 1970;49:2086-96.

${ }^{15}$ Quattrin N, Quattrin S, Cecio A. Nosographism of sea blue histiocytosis In: Seno S, Takaku F, Irino S. Topics in Haematology (Excerpta Medica International Congress Series, No. 415). Amsterdam: Excerpta Medica, 1977; 1036-9.

${ }^{16}$ Burns GF, Cawley JC, Flemans RJ, et al. Surface marker and other characteristics of Gaucher's cells $J$ Clin Pathol 1977;30:981-8.

Requests for reprints to: Dr PN Plowman, Department of Radiotherapy, St Bartholomew's Hospital, Smithfield, London EC1, UK. 